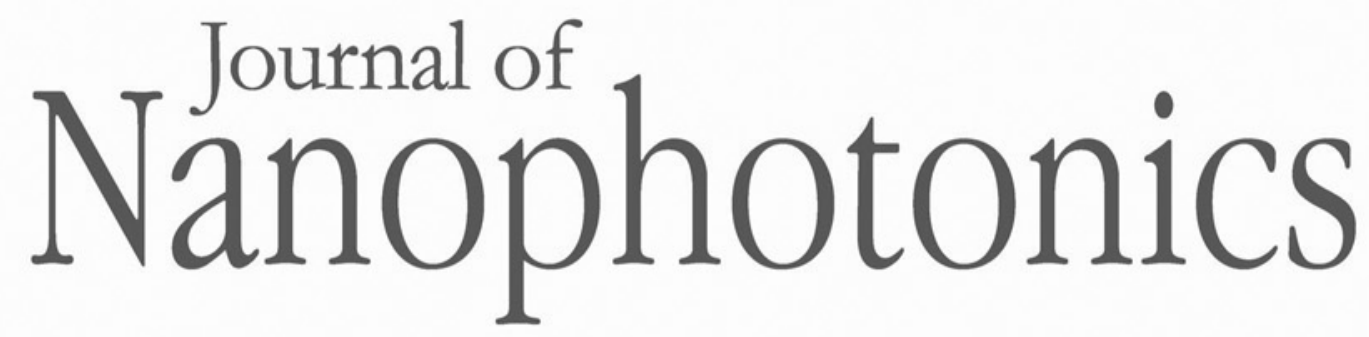

\title{
Photonic crystals with a continuous, Gaussian-type surface profile for near-perfect light trapping
}

Ping Kuang

Sayak Bhattacharya

Mei-Li Hsieh

Sajeev John

Shawn-Yu Lin 


\title{
Photonic crystals with a continuous, Gaussian-type surface profile for near-perfect light trapping
}

\author{
Ping Kuang, ${ }^{\text {a,* Sayak Bhattacharya, }}$ Mei-Li Hsieh, ${ }^{\mathrm{c}}$ Sajeev John, ${ }^{\text {b }}$ and \\ Shawn-Yu Lin ${ }^{\mathrm{a}}$ * \\ ${ }^{a}$ Rensselaer Polytechnic Institute, The Future Chips Constellation and the Department of \\ Physics, Applied Physics, and Astronomy, Troy, New York, United States \\ ${ }^{b}$ University of Toronto, Department of Physics, Toronto, Ontario, Canada \\ ${ }^{\mathrm{c}}$ National Chia-Tung University, Department of Photonics, Hsinchu, Taiwan
}

\begin{abstract}
We show a unique design of teepee-like photonic crystal (TP-PC) structure that possesses a true gradient, Gaussian-type surface profile for smooth and accurate index matching between air and silicon for near-perfect light trapping. Such funnel-like, inverse-conical topography is capable of achieving near-zero optical reflection and near-unity solar absorption with excellent angular response over the entire visible light wavelength range. The fabrication only requires standard microelectronics reactive-ion etching (RIE) process. We demonstrate how various process parameters, such as RIE gas mixture ratio, RIE power, thickness of silicon dioxide $\left(\mathrm{SiO}_{2}\right)$ coatings, and lattice constant of the photonic crystal, can impact the details of the "Gaussian" profile and further improve the optical performance of the TP-PC structure at broad- $\lambda$, broad- $\theta$, especially in the ultraviolet (UV) wavelength range. Our finite-difference time-domain (FDTD) simulation of the TP-PC structure reveals existence of multiple absorption resonances in the 800- to1100-nm wavelength range. Poynting vector plots show that such strong absorption enhancements at the resonant frequencies are due to long-lifetime photonic modes arising from parallel-to-interface refraction of the incoming sunlight and formation of vortex-like energy flow pattern inside the TP-PC structure. Our design will lead the way for future development of ultrathin, high-efficiency c-Si solar photovoltaics. (C) 2018 Society of Photo-Optical Instrumentation Engineers (SPIE) [DOI: 10.1117/1.JNP.12.026011]
\end{abstract}

Keywords: light trapping; antireflection; solar cells; ultrathin c-Si; reactive-ion etching; solar absorption.

Paper 17103 received Jul. 13, 2017; accepted for publication Apr. 30, 2018; published online May 21, 2018.

\section{Introduction}

Solar power provides sustainable, renewable, and clean energy resources. Solar photovoltaic (PV) market has been one of the fastest growing markets in recent years both globally and in the United States. ${ }^{1}$ However, the solar energy supply currently comprises of only less than $\sim 2 \%$ of the total energy consumption in the world. ${ }^{2}$ Various material systems have been explored for solar cell applications such as silicon, GaAs, CIGS, CdTe, perovskite, etc. ${ }^{3-7}$ However, the current PV market is still dominated by c-Si wafer-based solar PVs at $\sim 90 \%$ because of their great attributes such as being nontoxic, chemically stable, and abundant in nature. ${ }^{89}$ The current development and growth of c-Si solar PVs is constrained by the dollarper-Watt cost and materials supply. ${ }^{9-11}$ It is imperative to continue the fundamental research in cSi solar cells to develop silicon solar cell designs that are capable of near-perfect light trapping with ultrathin, high quality c-Si with its thickness at a cost-effective level $(\sim 10 \mu \mathrm{m})$ while trying to achieve world-record Si-based single-junction solar cell efficiency of approaching or greater than $30 \% .^{12-15}$

*Address all correspondence to: Ping Kuang, E-mail: ping.kuang@gmail.com; Shawn-Yu Lin, E-mail: sylin@ rpi.edu

$1934-2608 / 2018 / \$ 25.00$ (C) 2018 SPIE 
Previously, we have shown a unique design of teepee-like photonic crystal (TP-PC) structure, which possesses a true gradient, Gaussian-type surface profile for smooth and accurate index matching between air and silicon. ${ }^{16}$ Such funnel-like, inverse-conical topography is capable of achieving near-zero optical reflection and near-unity solar absorption with excellent angular response over the entire visible light wavelength range, an essential criterion for high-efficiency solar cells. The fabrication only requires standard microelectronics reactive-ion etching (RIE) process. In this work, we demonstrate how various process parameters, such as RIE gas mixture ratio, RIE power, thickness of silicon dioxide $\left(\mathrm{SiO}_{2}\right)$ coatings, and lattice constant of the photonic crystal, can impact the details of the "Gaussian" profile and further improve the optical performance of the TP-PC structure at broad- $\lambda$, broad- $\theta$, especially in the ultraviolet (UV) wavelength range. Our design will lead the way for future development of c-Si solar PVs with lower cost, being flexible, and achieving world-record Si-based single-junction solar cell efficiency of approaching or greater than $30 \%$.

Compare to traditional inverted pyramid structure by KOH wet etching, our TP-PC structure has much sharper trench angles, a truly continuous, nonlinear gradient-index surface profile. The height over pitch aspect-ratio $[h /(a / 2)]$ of the TP-PC structure is almost doubled than the inverted pyramid structure. In addition, such unique photonic structure design has superior light trapping capability in the weakly absorbing, near-infrared (IR) wavelength range for ultrathin c-Si $(10 \mu \mathrm{m})$ due to an optical phenomenon called "parallel-to-interface refraction" (PIR) effect, with which a near-normal incident light can be bent nearly 90 deg and much longer optical pathlength within the structure is achieved. ${ }^{17-20}$ Though the surface is slightly rougher due to RIE etching, such roughness can be mitigated by a simple removal process (shown in a later section). With silicon surface passivation processes, the surface recombination velocity will be significantly reduced and the effective minority carrier lifetime will be increased, which will enable c-Si solar cells with efficiency approaching and greater than $30 \% .^{21-25}$

\section{Methods}

To fabricate the TP-PC structures, we utilized a standard semiconductor fabrication process, which consists of a simple, one-step RIE etching on crystalline silicon to achieve the desired surface profile. First, photolithography was used to fabricate two-dimensional circular hole arrays with square symmetry on photoresist, which acts as the etch mask for the subsequent RIE etching process. DUV photoresists were spincoated on wafers, and exposure and patterning was done with ASML DUV stepper to achieve square lattice circular holes $(a=1.2 \mu \mathrm{m}$, $d=0.3 \mu \mathrm{m}$ ). Standard RIE etching (Oxford PlasmaLab 80+ RIE System) with $\mathrm{SF}_{6} / \mathrm{CHF}_{3}$ gas mixtures was used for silicon etch. $\mathrm{SiO}_{2}$ layer was grown by wet oxidation in an MRL Industries tube furnace at $900^{\circ} \mathrm{C}$ and then annealed at the same temperature. The thickness of $\mathrm{SiO}_{2}$ was characterized on a planar reference silicon piece using the FilMetrics (F50EXR) optical measurement system. For ultrathin TP-PC structures, silicon nitride $\left(\mathrm{S}_{3} \mathrm{~N}_{4}\right)$ was grown by low pressure chemical vapor deposition as the etch mask on the backside of silicon on insulator wafers first, then $12 \mathrm{~mm} \times 10 \mathrm{~mm}$ rectangular opening areas were patterned at the backside of the handle layer. $\mathrm{S}_{3} \mathrm{~N}_{4}$ in the opening areas was etched by RIE with $\mathrm{CHF}_{3} / \mathrm{O}_{2}$ recipe to expose the handle layer silicon. $\mathrm{KOH}(30 \%)$ wet etch was done at $80^{\circ} \mathrm{C}$ for $\sim 6 \mathrm{~h}$ to remove the handle layer silicon. The teepee photonic crystal on device layer was covered by an alkaline protective coating (ProTek-B3) for etch protection during $\mathrm{KOH}$ etch. Total absorption measurements were performed using an integrating sphere (Labsphere ${ }^{\circledR}$ ) with an unpolarized xenon or tungsten-halogen light source. Ocean Optics 2000+ spectrometer was used for data collection. The sample was inserted in the middle of the sphere and the absorption $(A)$ was measured. For angular dependence measurements, the sample holder was rotated accordingly with respect to the incident light beam.

\section{Results and Discussion}

Recent works of photonic nanostructures can offer very good antireflection property and light trapping for silicon solar cell applications. ${ }^{26-34}$ These nanoscale architectures also have 

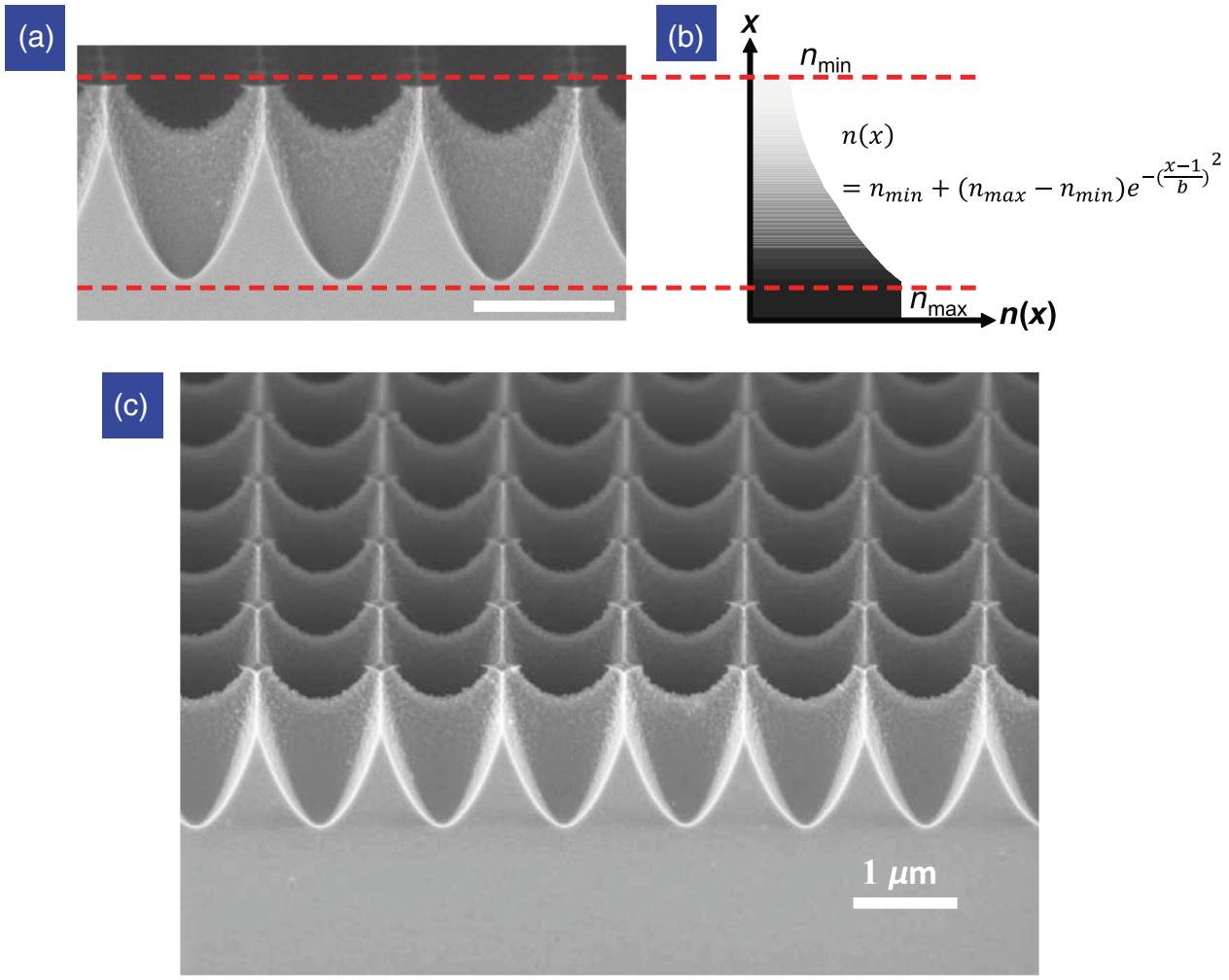

Fig. 1 (a) SEM image (side-view) shows the Gaussian-type profile of the teepee PC structure on c-Si. Scale bar $=1 \mu \mathrm{m}$. (b) Schematic representation of the refractive index profile of the Gaussian-type surface by the TP-PC structure. (c) SEM image of the TP-PC structure at slanted view.

outstanding angular response compared to regular quarter-wavelength ARC coatings. Furthermore, a unique design of gradient-index surface with a Gaussian-type profile was proposed by Chen et al. ${ }^{35}$ to show that a continuous, nonlinear surface profile facilitates smooth and accurate index matching between air and silicon, $n(x)$, to achieve almost zero reflection with excellent angular response. ${ }^{16,35}$ Such Gaussian-type surface profile can be expressed as

$$
n(x)=n_{\min }+\left(n_{\max }-n_{\min }\right) e^{-\left(\frac{x-1}{b}\right)^{2}},
$$

where $n_{\min }$ and $n_{\max }$ are the minimum and maximum refractive indices, respectively, $x$ is the normalized optical distance, which is proportional to the physical distance, and $b(\sim 0.52)$ is a fitting factor, which represents the shape width of the profile.

Figure 1(a) shows a side view of a TP-PC structure. It is clearly shown that the surface profile of the structure is continuous and smooth. It corresponds to $n(x)$ that the PC structure varies gradually along the vertical distance and the index change can be closely approximated by the Gaussian-type profile [Fig. 1(b)]. Such unique surface topography in c-Si will enable impinging lights preferentially and smoothly refracted by the sharp sidewall angles between the air/silicon interface and the backward reflection will be significantly minimized. Superior antireflection performance can be expected from our TP-PC structure.

Figure 1(c) shows the scanning electron microscopy (SEM) image of the teepee-like PC structure at bird-eye view. The structure shows a $\mathrm{V}$-shaped, inverse-conical like profile with very high $h /(a / 2)$ ratio $(\sim 2.3)$. The trench angle $\left(\theta_{\text {trench }}\right)$ is $\sim 50 \mathrm{deg}$ with very sharp trench bottoms, which is much steeper than that of an inverted pyramid structure profile obtained by $\mathrm{KOH}$ wet etching [trench angle $\sim 70 \mathrm{deg}, h /(a / 2)$ ratio $\sim 1.3$ ]. We expect our TP-PC structure will have much better light trapping capability due to such greater vertical depth and steeper sidewall angles, along with the continuous, gradually changed surface profile. It should be noted that some surface roughness is introduced during the RIE dry etch process, which may lead to 

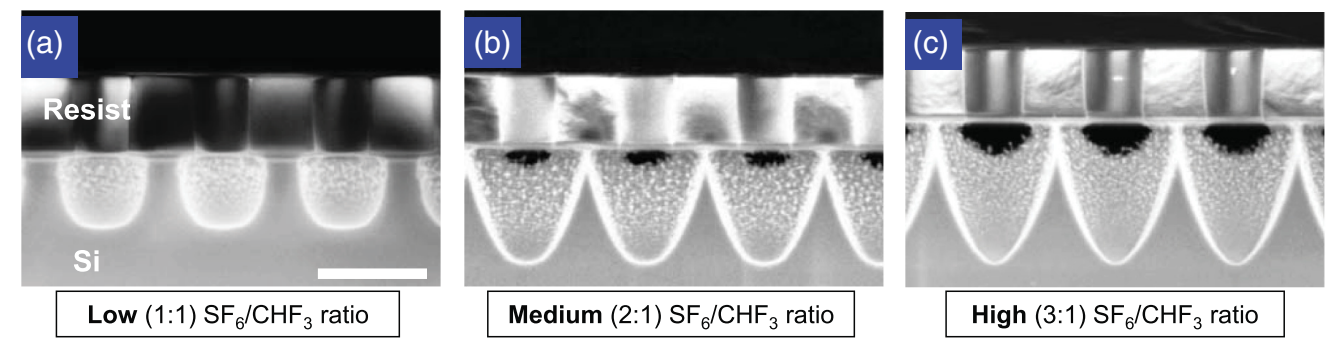

(d)
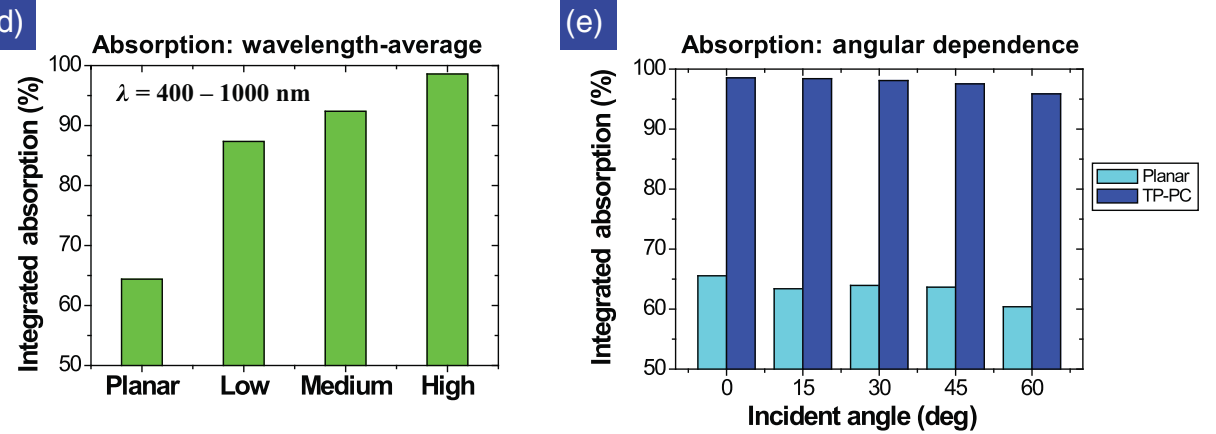

Fig. 2 Mixed-gas-ratio dependence of the etched profile. SEM images (cross-sectional view) of the PC structures fabricated at (a) low $\mathrm{SF}_{6} / \mathrm{CHF}_{3}$ ratio, (scale bar $=1 \mu \mathrm{m}$ ), (b) medium $\mathrm{SF}_{6} / \mathrm{CHF}_{3}$ ratio, and (c) high $\mathrm{SF}_{6} / \mathrm{CHF}_{3}$ ratio, resulting in a teepee-like $\mathrm{PC}$ structure (all samples with RIE power $=30 \mathrm{~W}$, pressure $=30 \mathrm{mTorr}$ ). (d) Measured integrated absorption ( $\lambda=400$ to $1000 \mathrm{~nm})$ of four different samples [planar silicon reference $(t=500 \mu \mathrm{m})$, RIE with low, medium, and high $\mathrm{SF}_{6} / \mathrm{CHF}_{3}$ ratio, respectively]. (e) Measured angular dependence of the integrated absorption for planar silicon reference and the TP-PC structure with high $\mathrm{SF}_{6} / \mathrm{CHF}_{3}$ ratio.

surface defects and high surface recombination velocity on the silicon surface. ${ }^{21}$ Such issues can be mitigated by high-temperature oxidation processes, which will be discussed in a later section.

Fluorine-based gas chemistry $\left(\mathrm{SF}_{6}\right.$ and $\left.\mathrm{CHF}_{3}\right)$ was used for the RIE etching at relative low power and chamber pressure to avoid excessive surface roughness and defects on the silicon surface. First, the etching process was carried out at different $\mathrm{SF}_{6} / \mathrm{CHF}_{3}$ ratios to examine its effect on the surface profile. It had been shown that $\mathrm{SF}_{6}$ and $\mathrm{CHF}_{3}$ gas mixture-based RIE etching can produce certain sidewall angles and undercut in c-Si patterning process, but the trench bottoms still remain flat. ${ }^{36}$ Here, we show that, in addition to desired sidewall angles and undercut, nonlinear, continuously changed sidewall angles and sharp trench bottoms can also be achieved by our RIE etching approach. As shown in Fig. 2(a), when the low (1:1) $\mathrm{SF}_{6} / \mathrm{CHF}_{3}$ gas ratio was used, $\mathrm{CHF}_{3}$ generates sufficient organic passivation layer on the silicon to suppress the aggressive $\mathrm{SF}_{6}$ etching reaction with silicon and promote slower, anisotropic etching, which results in shallow etch depth, flat trench bottoms, and vertical sidewalls. As the medium (2:1) $\mathrm{SF}_{6} / \mathrm{CHF}_{3}$ gas ratio was used [Fig. 2(b)], more $\mathrm{SF}_{6}$ gas was supplied and fluorine-based radicals were able to facilitate faster and isotropic etching in the lateral direction. This resulted in slanted sidewalls and rounded trench bottoms. Furthermore, a slight undercut opening at the top was obtained between each adjacent silicon etching profile. Finally, when the $\mathrm{SF}_{6} / \mathrm{CHF}_{3}$ gas ratio was increased to 3:1, further improvement was observed, with much sharper sidewall angles and trench bottoms [Fig. 2(c)]. The etch rates were increased in both vertical and lateral directions and deeper etch depth and larger undercut opening were resulted. This structure coincidentally follows the aforementioned Gaussian-like surface profile with continuous, nonlinear, gradually changed sidewalls, which also closely resembles a V-shaped, inverted-conical structure. Therefore, this RIE etching process provides a simple and fast approach capable of realizing nanostructures with various surface profiles.

The wavelength-averaged total integrated absorption $\left(A_{\text {avg }}\right)$ of the samples etched with different $\mathrm{SF}_{6} / \mathrm{CHF}_{3}$ gas ratios was measured for wavelength range $\lambda=$ [400 to $1000 \mathrm{~nm}$. As described in a later section, photoresist was removed and surface roughness was reduced, and a 60-nmthick $\mathrm{SiO}_{2}$ was grown on the structure before the measurement. For comparison, the absorption 

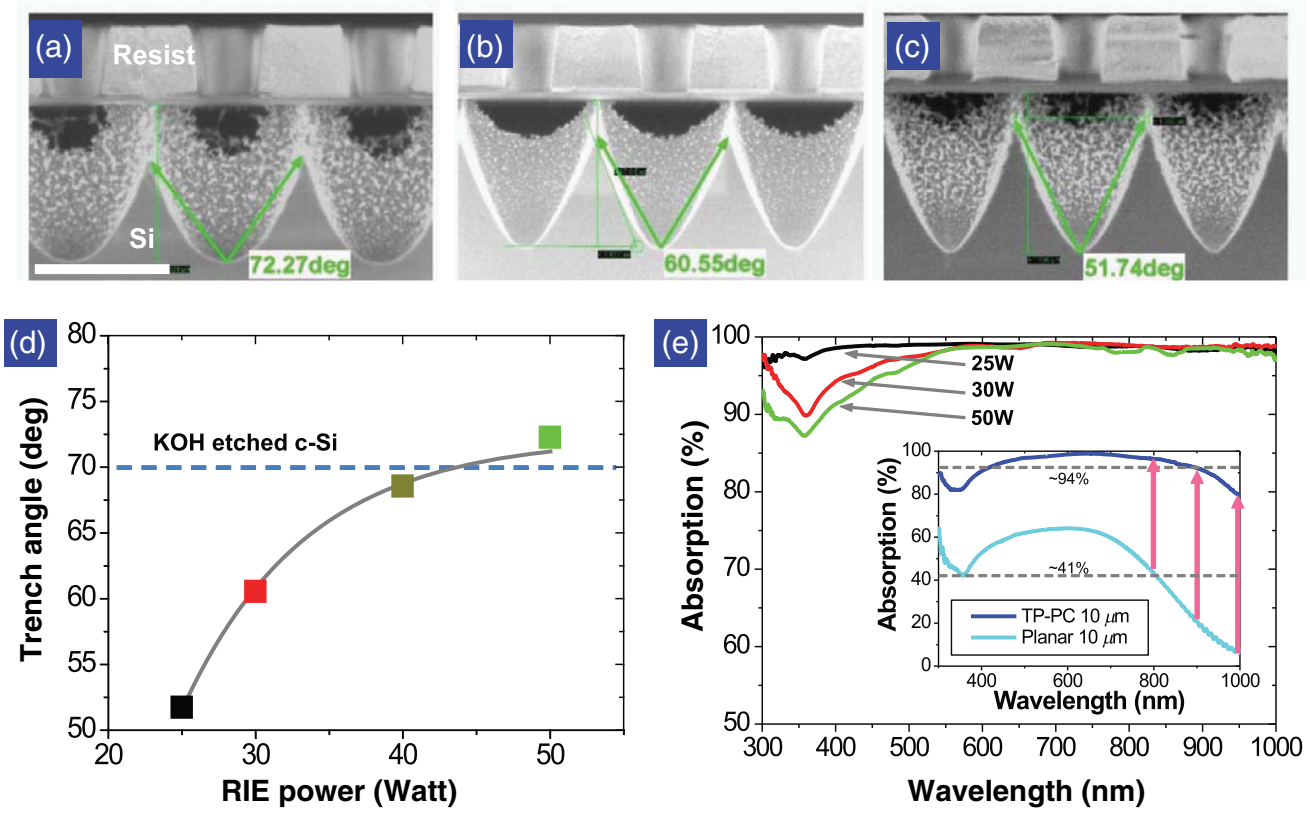

Fig. 3 RIE power dependence of the etched profile. SEM images (cross-sectional view) of the PC structures fabricated at (a) RIE power $=50 \mathrm{~W}$ (scale bar $=1 \mu \mathrm{m}$ ), (b) RIE power $=30 \mathrm{~W}$, and (c) RIE power $=25 \mathrm{~W}$, resulting in a much sharper trench angles in the teepee-like PC structure (30 mTorr, 3:1 $\mathrm{SF}_{6} / \mathrm{CHF}_{3}$ ratio). (d) Dependence of the trench angles to the RIE power. Dashed blue line: trench open angle for a typical $\mathrm{KOH}$ wet etched inverted pyramid structure. (e) Measured absorption of three samples with different RIE powers (black: 25-W RIE power, red: 30-W RIE power, and green: 50-W RIE power) shows that at lower RIE power and smaller trench opening angle, the solar absorption in the near-UV range $(\lambda=300$ to $600 \mathrm{~nm})$ was increased. Also shown in (e) inset is the measured absorption for a TP-PC structure on ultrathin silicon $(t=10 \mu \mathrm{m}) .{ }^{16}$

of a planar silicon $(t=500 \mu \mathrm{m})$ was also taken [Fig. 2(d)]. The planar silicon reference has an expected $A_{\text {avg }}$ of $65 \%$. The averaged absorption is $87 \%, 92 \%$, and $99 \%$ for low, medium, and high $\mathrm{SF}_{6} / \mathrm{CHF}_{3}$ gas ratio etched samples, respectively. The near-unity absorption (99\%) for the high (3:1) $\mathrm{SF}_{6} / \mathrm{CHF}_{3}$ gas ratio etched sample is among the highest that have been achieved such as black-silicon. ${ }^{37}$ Evidently, the RIE etched samples all have better absorption, and it is further improved when the $\mathrm{SF}_{6} / \mathrm{CHF}_{3}$ ratio is increased. The optical absorption of the RIE etched samples is directly related to the surface profile of the structures. The high $\mathrm{SF}_{6} / \mathrm{CHF}_{3}$ ratio etched structure, the TP-PC, is Gaussian-like, and near-unity absorption is achieved. Therefore, the excellent antireflection property of the Gaussian-type surface profile with the gradually changed index at the air-Si interface is the main cause for the near-unity absorption.

Furthermore, the angular dependence of the integrated absorption $\left(\theta_{\text {inc }}=0\right.$ deg to $\left.60 \mathrm{deg}\right)$ for the TP-PC structure by high $\mathrm{SF}_{6} / \mathrm{CHF}_{3}$ ratio was plotted as well as for the planar silicon [Fig. 2(e)]. The plot shows that the decrease of absorption is negligible for $\theta_{\text {inc }}=0$ deg to $45 \mathrm{deg}$. At the maximum angle of incidence $\theta_{\text {inc }}=60 \mathrm{deg}$, the averaged absorption is still $\sim 96 \%$, which is also among the highest that have been achieved. ${ }^{37}$ However, for the $500-\mu \mathrm{m}$-thick planar c-Si, the absorption is dropped noticeably to $\sim 60 \%$ at $\theta_{\text {inc }}=60 \mathrm{deg}$. The outstanding angular response of the TP-PC structure is beneficial for solar light collections even at large angles of incidence, which fulfills one of the critical requirements for enabling highefficiency c-Si solar cell applications.

In order to further improve light trapping by the TP-PC structure, the effect of RIE power on the etched profile of the TP-PC structures was studied. The RIE power was varied between 25 and $50 \mathrm{~W}$ while other parameters remained the same as for the high $\mathrm{SF}_{6} / \mathrm{CHF}_{3}$ ratio. It is clearly shown, from the SEM images [Figs. 3(a)-3(c)], that as the RIE power is reduced, the trench angle $\left(\theta_{\text {trench }}\right)$ becomes sharper. It should be noted that, in order to achieve the same etch depth, longer etch time was required for lower RIE power because the etch rate was slower at lower RIE power. However, the total etch energy (power $\times$ time) was the same for each 
case to reach the same etch depth. It is believed that, at lower RIE power, less fluorine radicals are present during etching. Therefore, the etching was less isotropic in the lateral direction. Also, since the fluorine ions are less energetic, the etch rate is slower. Figure 3(d) shows a plot of the trench angles at different RIE powers. For TP-PC structure obtained with RIE power $=50 \mathrm{~W}$, $\theta_{\text {trench }}$ is $\sim 72.3 \mathrm{deg}$. When the RIE power is reduced to $40 \mathrm{~W}, \theta_{\text {trench }}$ is $\sim 68.6 \mathrm{deg}$. The trench angle is further reduced to $\sim 60.6 \mathrm{deg}$ when RIE power is $30 \mathrm{~W}$. Finally, when the minimum RIE power of $25 \mathrm{~W}$ was used, the trench angle is only $\sim 51.7 \mathrm{deg}$. This indicates that TP-PC structure with trench angle smaller than that of the inverted pyramid can be realized by RIE etching process at a range of RIE powers. For reference, the trench angle for a $\mathrm{KOH}$-etched inverted pyramid is $\sim 70 \mathrm{deg}$, which is fixed and dictated by the $\langle 111\rangle$ crystalline plane of silicon (dashed line). The results show that, compared to the standard silicon wet-etch process, RIE etching offers a superior and more flexible, controllable route for creating sharper trench angles in c-Si nanostructured patterns.

The trench open angle has great influence on the solar light absorption, especially in the nearUV wavelength range. As shown in Fig. 3(e), different RIE powers all result in very high absorption $(A \sim 99 \%)$ in the longer wavelength range $(\lambda=600$ to $1000 \mathrm{~nm})$. As the RIE power is reduced and the trench angle gets smaller, the absorption of the TP-PC structure in the shorterwavelength and near-UV range $\left(\lambda=300\right.$ to $600 \mathrm{~nm}$ ) is increased. The $A_{\text {avg }}$ is $\sim 93.8 \%$ when RIE power is $50 \mathrm{~W}$ for $\lambda=300$ to $600 \mathrm{~nm}$. It is further increased to $\sim 95.8 \%$ at RIE power $=30 \mathrm{~W}$. The average absorption keeps increasing, eventually reaching $\sim 98.5 \%$ for 25 -W RIE etched sample. This indicates that sharper trench is capable of trapping more of the shorter-wavelength light, especially the near-UV light. This is due to enhanced optical resonances for shorterwavelength light in the sharper trench regions, promoting long photon dwell time and higher probability of being absorbed. Therefore, in addition to the Gaussian-type surface profile of the TP-PC structure, further enhanced light trapping by sharper trench open angles can provide additional improvement in solar cell efficiency of the c-Si PVs. This phenomenon could have a significant impact for near-UV light trapping for other applications, such as photocatalysis, as well.

Also shown in Fig. 3(e) inset is the absorption of the TP-PC structure on a $10-\mu \mathrm{m}$, ultrathin c-Si, with a 60-nm $\mathrm{SiO}_{2}$ front surface ARC coating and 200-nm Ag backside reflector, for $\lambda=$ [300 to $1000 \mathrm{~nm}$ ]. The maximum absorption is at $\lambda \sim 650 \mathrm{~nm}$ and is close to unity $\left(A_{\max } \sim 98.8 \%\right)$. Also shown in Fig. 3(e) inset is the measured absorption of a 10- $\mu \mathrm{m}$-thick planar c-Si, which shows an $A_{\max } \sim 64.1 \%$ at $\lambda \sim 600 \mathrm{~nm}$. The measured absorption is gradually decreased to $\sim 42 \%, \sim 20 \%$, and $\sim 6 \%$ at $\lambda=800,900$, and $1000 \mathrm{~nm}$, respectively, in the near-IR region due to the weak absorption at the silicon bandgap edge. On the contrary, the absorptions are sustained at high levels for the 10- $\mu \mathrm{m}$ TP-PC Si structure, at the same wavelengths, which are $\sim 96 \%, \sim 90 \%$, and $\sim 79 \%$, respectively. The absorption enhancements are $\sim 2.3, \sim 4.5$, and $\sim 13$ times, respectively, for the TP-PC structure compared to the planar silicon. The enhancement is a direct consequence of the PIR effect for improved light trapping with nearly perpendicular light-bending and increased optical path lengths phenomenon in the unique photonic crystal structure on an ultrathin c-Si. Furthermore, the average absorption $\left(A_{\text {avg }}\right)$ of the $10-\mu \mathrm{m}$ planar silicon is $41.2 \%$ for $\lambda=$ [400 to $1000 \mathrm{~nm}$ ], and $A_{\text {avg }}$ for $10-\mu \mathrm{m}$ silicon TP-PC is $\sim 94.7 \%$, which, to our knowledge, is the highest absorption ever achieved for a $10-\mu \mathrm{m}$ ultrathin $\mathrm{c}-\mathrm{Si}$. The total average absorption enhancement of $10-\mu \mathrm{m}$ TP-PC over planar silicon is also $\sim 2.3$. This indicates that, with only $10 \mu \mathrm{m}$ of the silicon material, a significant portion of the near-IR solar irradiance can be harnessed. Therefore, our unique TP-PC structure offers a new route for improving the solar energy collection and conversion efficiency of ultrathin c-Si solar cells.

It should be noted that the RIE etching for TP-PC fabrication results in a slightly rougher surface on c-Si. It is necessary to mitigate such surface roughness, especially for achieving higher c-Si surface quality with minimal surface recombination for better electrical performance in PV devices. Figure 4 shows the process for the surface roughness removal. After RIE etching [Fig. 4(a)], photoresist on the sample was dry stripped by $\mathrm{O}_{2}$ plasma for $10 \mathrm{~min}$ at an RIE power $=200 \mathrm{~W}$, pressure $=200 \mathrm{mTorr}$, and $\mathrm{O}_{2}$ gas flow $=50 \mathrm{sccm}$. Figure $4(\mathrm{~b})$ shows that there still were significant amount of organic residue leftovers on top of the silicon after the $\mathrm{O}_{2}$ plasma dry strip. In order to completely remove such organic residues, wet oxidation was carried out at $900^{\circ} \mathrm{C}$ for $20 \mathrm{~min}$ (MRL Industries tube furnace). It is clearly shown that the hightemperature oxidation step removed the organic leftovers completely, and a thin $\mathrm{SiO}_{2}$ layer was 

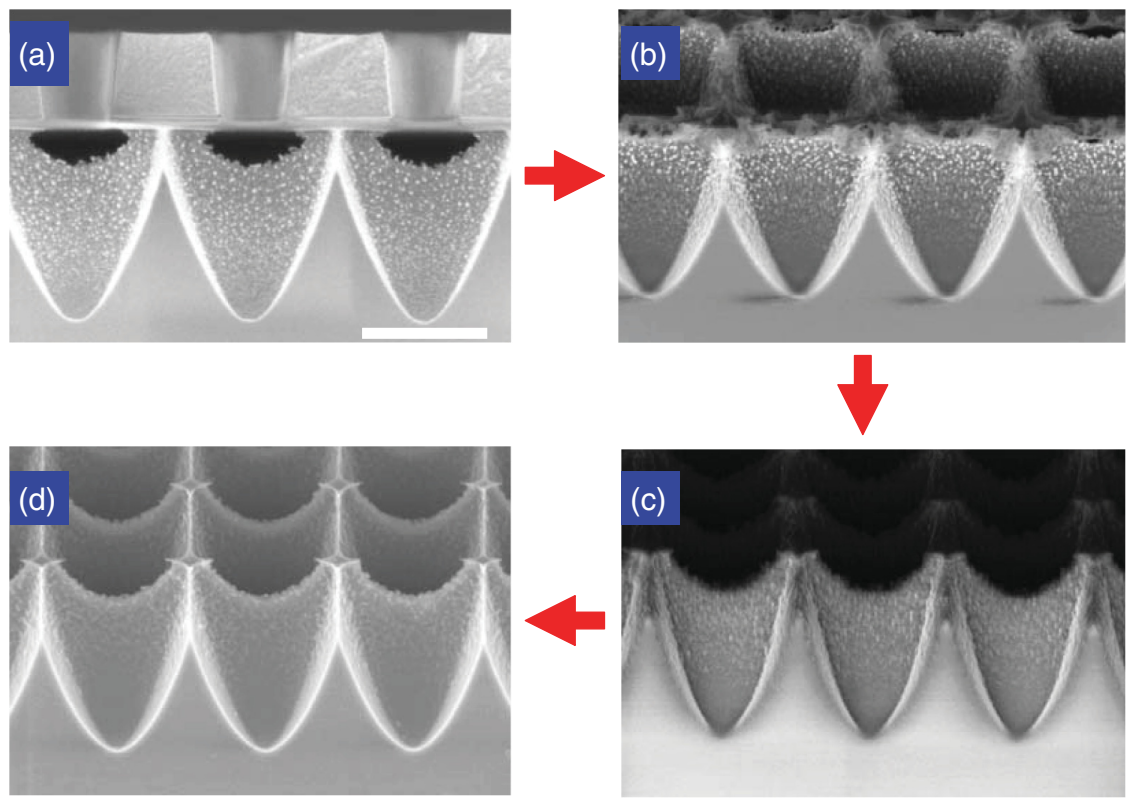

Fig. 4 Illustration of process for surface roughness removal. SEM images (cross-sectional view) of the TP-PC structure (a) after c-Si RIE etch, (b) after $\mathrm{O}_{2}$ plasma PR dry strip, (c) after high-temp wet oxidation $\left(T=900^{\circ} \mathrm{C}\right)$, and $(\mathrm{d})$ after oxide removal by $\mathrm{BOE}(6: 1)$ (scale bar: $\left.1 \mu \mathrm{m}\right)$.
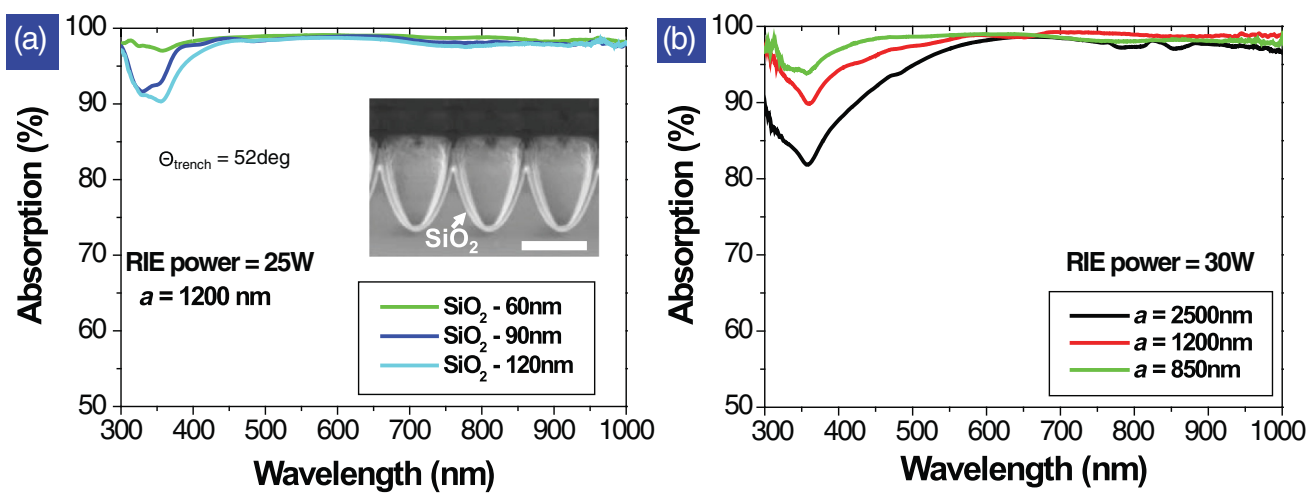

Fig. 5 (a) Dependence on $\mathrm{SiO}_{2}$ ARC-coating thickness. Measured absorption of TP-PC structure $\left(a=1200 \mathrm{~nm}\right.$ ) with different $\mathrm{SiO}_{2}$-coating thicknesses (green: $60 \mathrm{~nm}$, blue: $90 \mathrm{~nm}$, cyan: $120 \mathrm{~nm}$ ). It shows that with thinner $\mathrm{SiO}_{2}$, the solar absorption in the near-UV range ( $\lambda=300$ to $450 \mathrm{~nm}$ ) was noticeably increased. Inset: SEM image of the TP-PC structure with 120-nm $\mathrm{SiO}_{2} \mathrm{Coating}$ (scale bar $=1 \mu \mathrm{m}$ ). (b) Dependence on PC lattice constant. Measured absorption of TP-PC structures with different lattice constants (green: $a=850 \mathrm{~nm}$, red: $1200 \mathrm{~nm}$, black: $2500 \mathrm{~nm}$ ). It shows that with smaller lattice constant, the solar absorption in the near-UV range $(\lambda=300$ to $600 \mathrm{~nm})$ was noticeably increased.

grown on the silicon surface [Fig. 4(c)]. Subsequently, the sample was placed in a 6:1 buffered oxide etch (BOE) solution for $\sim 3$ min to remove the oxide layer. Figure 4(d) shows that, after BOE (6:1), the oxide layer was completed etched away and the final TP-PC structure is revealed. Compared to the original TP-PC structure right after RIE etching, the final structure possesses a c-Si surface with much less surface roughness. In addition, the structural mechanical integrity and surface profile of the TP-PC structure are maintained after several steps of surface modification. It is necessary to mention that, for the PV device fabrication, additional surface passivation coatings such as $\mathrm{Al}_{2} \mathrm{O}_{3}$ or $\mathrm{SiO}_{2}$ can be applied to further reduce the surface recombination. ${ }^{22-24,37,38}$ 
It had been shown that $\mathrm{SiO}_{2}$ coating on c-Si patterned structures can provide excellent antireflection property as well as great surface passivation. ${ }^{22-24,38}$ In order to understand the effect of the $\mathrm{SiO}_{2}$ ARC-coating thickness on the absorption of TP-PC structures, $\mathrm{SiO}_{2}$ layer was grown by the aforementioned wet oxidation process at $900^{\circ} \mathrm{C}$ at various times to achieve different thicknesses of 60,90 , and $120 \mathrm{~nm}$ for the TP-PC structure $(500-\mu \mathrm{m}$-thick c-Si, lattice constant $=1200 \mathrm{~nm}$, and RIE power $=25 \mathrm{~W}$ ). It is observed that, for the majority of the whole visible light spectrum $(\lambda=400$ to $1000 \mathrm{~nm})$, the absorption is extremely high at $\sim 98 \%$ to $99 \%$ [Fig. 5(a)]. All three samples have similarly flat spectra in this wavelength range, indicating highly efficient and near-unity light trapping. Interestingly, in the near-UV wavelength range, the absorption is increased with decreasing $\mathrm{SiO}_{2}$ thickness. The average absorption for $\lambda=$ [300 to $400 \mathrm{~nm}$ ] is $92.9 \%, 94.6 \%$, and $97.7 \%$ for $\mathrm{SiO}_{2}$ thicknesses of 120,90 , and $60 \mathrm{~nm}$, respectively. It is evident that thinner $\mathrm{SiO}_{2}$ can better enhance the solar absorption in the UV wavelength range. This is because thinner $\mathrm{SiO}_{2}$ suppresses the optical reflection in the UV wavelength range more efficiently and provides better index-matching between the air and $\mathrm{Si}$ interface. Figure 5 inset shows a SEM image of the TP-PC structure with $120-\mathrm{nm} \mathrm{SiO}_{2}$ coating. It is clearly shown that the wet oxidation creates a conformal coating on the high aspect-ratio TP-PC structure, especially in the trench regions. The surface roughness has been reduced noticeably. Therefore, our ultrathin $\mathrm{SiO}_{2}$ by wet oxidation process can also provide a similar, if not superior, surface passivation purpose.

One of the fundamental characteristics of photonic crystals is the scaling property with regard to optical spectrum. Therefore, it is important to understand the effect of lattice constant on the absorption for the TP-PC structures. TP-PC structures with three different lattice constants, $a=850,1200$, and $2500 \mathrm{~nm}$, were fabricated by standard RIE etching process on $500-\mu \mathrm{m}$-thick c-Si wafers (RIE power $=30 \mathrm{~W}, 60$-nm-thick $\mathrm{SiO}_{2}$ ARC). The absorption spectra [Fig. 5(b)] show that the optical absorption of TP-PC structure increases with decreasing lattice constant. It is clear that for lattice constant $a=2500 \mathrm{~nm}$, the absorption is the lowest, with a dip at $\lambda \sim 355 \mathrm{~nm}$. The overall absorption is lower for $a=2500 \mathrm{~nm}$ compared to other two lattice constants. Furthermore, as the lattice constant is decreased, the dip at $\lambda \sim 355 \mathrm{~nm}$ becomes less pronounced. For $\lambda=$ [300 to $600 \mathrm{~nm}$ ], the $A_{\text {avg }}$ is $97.5 \%, 95.8 \%$, and $91.3 \%$ for $a=850,1200$, and $2500 \mathrm{~nm}$, respectively. Therefore, TP-PCs with smaller lattice constants have higher solar absorption. The dependence of absorption on the lattice constant is currently under study, but it is clear that the enhanced absorption is due to increased resonance modes in the TP-PC with smaller lattice constants to allow better light trapping, especially for shorter-wavelength near-UV light. Therefore, TP-PC structures with smaller lattice constant can further improve the absorption enhancement for solar PV applications.

Figure 6 shows the results of our finite-difference time-domain (FDTD) computations for the $10-\mu \mathrm{m}$-thick TP-PC structure. Figure 6(a) compares the energy density distributions in a $10-\mu \mathrm{m}$ planar c-Si slab (with 200-nm Ag backreflector) and 10- $\mu$ m TP-PC (with 200-nm Ag backreflector and $60-\mathrm{nm} \mathrm{SiO}_{2} \mathrm{ARC}$ ) corresponding to absorption resonances at $\lambda=810,903$, and $1000 \mathrm{~nm}$. For a given wavelength, the energy density distributions in both the structures are normalized by the average energy density within the planar c-Si slab at that wavelength and we define energy density enhancement factor at a given point as the ratio of the energy density at that point to the normalization factor. At $\lambda=810 \mathrm{~nm}$, the dominant mechanism responsible for absorption enhancement is the creation of high concentration electric field regions (with energy density $\sim 12$ times than that in planar silicon) near the tip of the TP-PC structure. As the magnified view of the Poynting vector plot in Fig. 6(b) shows, the normally incident energy is bent by $90 \mathrm{deg}$ and enters the structure almost horizontally. Although this mechanism of creation of high concentration field regions near the tip is present for resonances at 903 and $1000 \mathrm{~nm}$ as well, the horizontal entrance of light near the tip is more pronounced at $810 \mathrm{~nm}$. Figures 6(c) and 6(d) show magnified views of the lower half of the TP-PC structure at 903 and $1000 \mathrm{~nm}$, respectively. The Poynting vector plot in the highlighted parts of Fig. 6(c) reveals vortex-like circulation pattern in the energy flow. These vortices are formed between the regions of high field concentration (with energy densities $\sim 16$ to 22 times higher than that of planar silicon). As we move to the resonance at $1000 \mathrm{~nm}$, we find that the energy density enhancement factor at the hot spots inside the structure reaches a maximum value of $\sim 17$ [shown in Fig. 6(d)]. This resonance is attributed to the formation of vortex-like energy circulation pattern between the hot spots as 

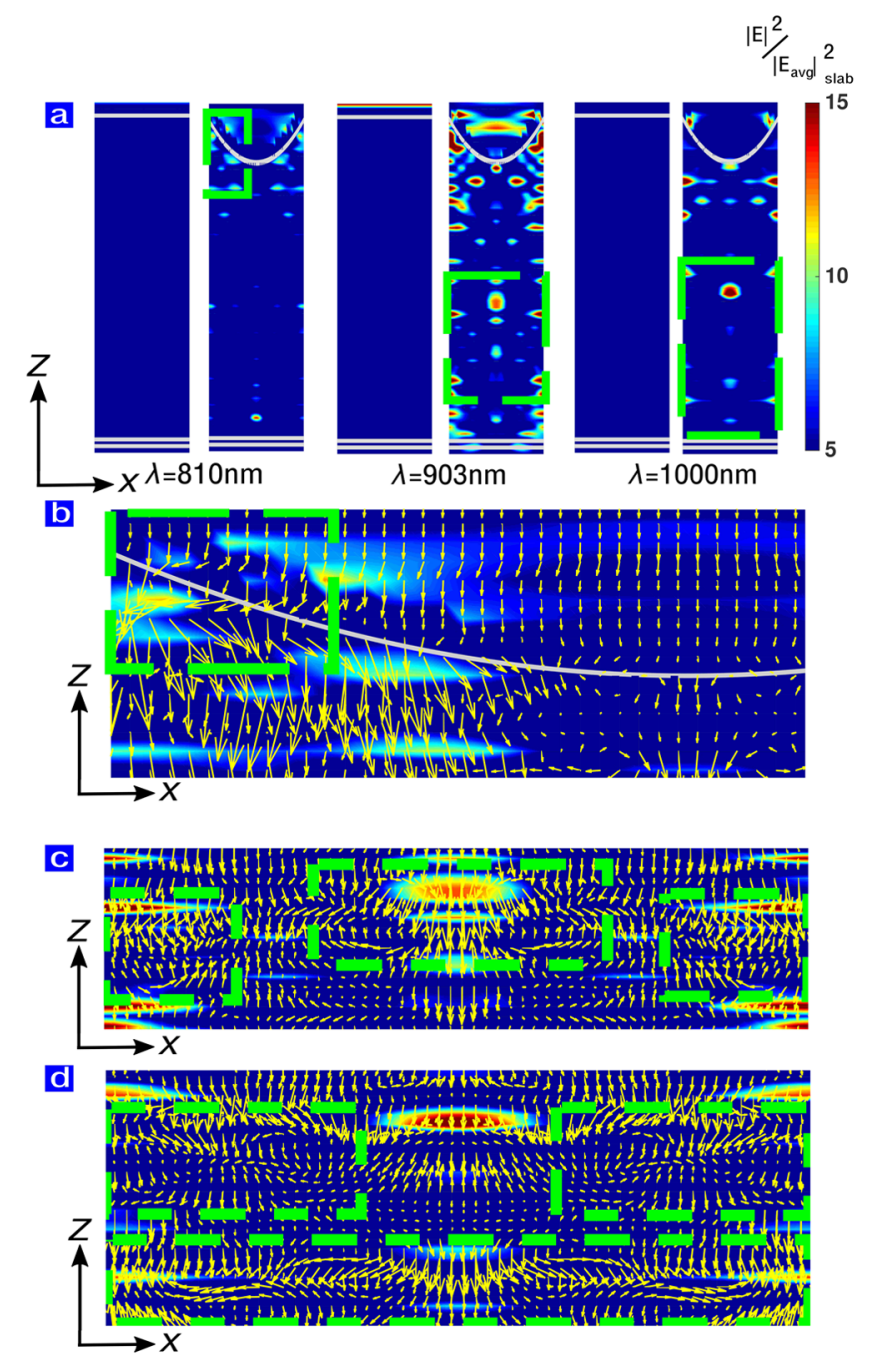

Fig. 6 Ultrathin Si with TP-PC structure. Figure 1 (a) calculated energy density distribution over the cross sections of 10-m-thick planar silicon (200-nm Ag backreflector) and 10- $\mu \mathrm{m}$ TP-PC structure (200-nm Ag backreflector, 60-nm $\mathrm{SiO}_{2} \mathrm{ARC}$ ) for resonances at $\lambda=810,903$, and $1000 \mathrm{~nm}$, respectively. The TP-PC structure is periodic in $x y$-plane and the wave vector of the incident plane wave is directed along - z-axis. Planar Si surface, parabolic profile, and the position of the Ag backreflector are shown by gray continuous lines. The energy densities are normalized by $\left|E_{\text {avg }}\right|_{\text {slab }}^{2}$, the average energy density in 10- $\mu \mathrm{m}$ planar silicon. (b), (c), and (d) are magnified views of highlighted regions of (a), showing the slices of the in-plane Poynting vector for $\lambda=810,903$, and $1000 \mathrm{~nm}$, respectively. The highlighted region of (b) corresponds to the region near the tip of the TP-PC structure and shows almost parallel to interface entrance of normally incident light $(\lambda=810 \mathrm{~nm})$. The parabolic profile has been shown by the gray continuous line. The highlighted regions of (c) show vortex-like circulation pattern of the Poynting vector. The highlighted regions in the upper part of (d) show similar vortex formation at $1000 \mathrm{~nm}$. The lower highlighted region of (d) shows parallel to interface energy flow in addition to vortex-like Poynting vector.

well as parallel to interface energy flow [shown in the highlighted parts of Fig. 6(d)]. Both vortex-like circulation and parallel to interface flow of energy increase the dwell time of the photons inside the TP-PC structure and thus, increase the absorption in 800- to 1100-nm wavelength range.

\section{Conclusion}

In conclusion, we have realized a unique design of TP-PC structure capable of broadband, wideangle near-unity solar absorption with a true gradient, Gaussian-type surface profile for smooth, 
and accurate index matching at the air/Si interface to achieve almost zero reflection with excellent angular response. We show that desired TP-PC structures can be achieved by optimizing the dry etch parameters such as gas-mixture ratio and RIE etching powers and by optimizing the ARC-coating thickness and the lattice constant. The optimal structures show near-unity solar absorption for a very broad wavelength range $(\lambda=300$ to $1000 \mathrm{~nm})$. Our design will lead the way for future development of c-Si solar PVs, particularly the ultrathin c-Si PVs, with lower cost, being flexible, and achieving world-record Si-based single-junction solar cell efficiency of approaching or greater than $30 \%$.

\section{Simulation Methodology}

Our FDTD calculations are performed using electromagnetic template library. ${ }^{39}$ Periodic boundary conditions are applied along $x$ - and $y$-directions. The computation boundaries, normal to $z$-direction, are terminated with perfectly matched layers. A broadband plane wave, containing significant energy in the 300- to 1100 -nm wavelength range, is assumed to illuminate the photonic crystal from $+z$-direction. A flux plane placed between the TP-PC structure and the top boundary of the computation box measures the reflection $(R)$ from the structure. Similarly, the transmission $(T)$ is measured by another flux plane placed between the Ag backreflector and the computation box boundary at the bottom. Since the 100-nm Ag backreflector has negligible parasitic absorption, ${ }^{19,40,41}$ the total absorption $A(\lambda)$ in the $\mathrm{c}$-Si layer is given by $A(\lambda)=1-R(\lambda)-T(\lambda)$.

\section{Acknowledgments}

S.Y.L. and S.J. gratefully acknowledge financial support from DOE-BES under award DEFG02-06ER46347. M.L.H. acknowledges partial financial support from NSC 101-2221-E009-110 and also travel support from DOE-BES for international collaboration. P.K. gratefully acknowledges fabrication assistance by Anthony Post and valuable discussions with Vince Genova. This work was performed in part at the Cornell NanoScale Facility, a member of the National Nanotechnology Coordinated Infrastructure (NNCI).

\section{References}

1. A. Hobson and M. Munsell, "U.S. solar market grows 95\% in 2016, smashes records," http://www.seia.org/news/us-solar-market-grows-95-2016-smashes-records (14 February 2017).

2. International Energy Agency, "Key world energy statistics 2016," http://www.iea.org/ publications/freepublications/publication/KeyWorld2016.pdf (4 May 2017).

3. M. A. Green et al., "Solar cell efficiency tables (Version 49)," Prog. Photovoltaics Res. Appl. 23, 1-9 (2017).

4. K. Yoshikawa et al., "Silicon heterojunction solar cell with interdigitated back contacts for a photoconversion efficiency over 26\%," Nat. Energy 2, 17032 (2017).

5. Solar Frontier Press Release, "Solar frontier achieves world record thin-film solar cell efficiency: 22.3\%," http://www.solar-frontier.com/eng/news/2015/C051171.html (8 December 2015).

6. First Solar Media, "First solar achieves yet another cell conversion efficiency world record," http://www.businesswire.com/news/home/20160223005315/en/ (23 February 2016).

7. W. S. Yang et al., "High-performance photovoltaic perovskite layers fabricated through intramolecular exchange," Science 348, 1234-1237 (2015).

8. A. Blakers et al., "High efficiency silicon solar cells," Energy Procedia 33, 1-10 (2013).

9. N. S. Lewis, "Research opportunities to advance solar energy utilization," Science 351, aad1920 (2016).

10. A. Goodrich et al., "A wafer-based monocrystalline silicon photovoltaics road map: utilizing known technology improvement opportunities for further reductions in manufacturing costs," Sol. Energy Mater. Sol. Cells 114, 110-135 (2013). 
11. D. M. Powell et al., "Crystalline silicon photovoltaics: a cost analysis framework for determining technology pathways to reach baseload electricity costs," Energy Environ. Sci. 5, 5874-5883 (2012).

12. M. S. Branham et al., "15.7\% efficient $10-\mu \mathrm{m}$-thick crystalline silicon solar cells using periodic nanostructures," Adv. Mater. 27, 2182-2188 (2015).

13. S. Eyderman et al., "Light-trapping optimization in wet-etched silicon photonic crystal solar cells," J. Appl. Phys. 118, 023103 (2015).

14. S. Jeong, M. D. McGehee, and Y. Cui, "All-back-contact ultra-thin silicon nanocone solar cells with 13.7\% power conversion efficiency," Nat. Commun. 4, 2950 (2013).

15. G. Li et al., "Nanopyramid structure for ultrathin c-Si tandem solar cells," Nano Lett. 14, 2563-2568 (2014).

16. P. Kuang et al., "Achieving an accurate surface profile of a photonic crystal for near-unity solar absorption in a super thin-film architecture," ACS Nano 10, 6116-6124 (2016).

17. A. Chutinan and S. John, "Light trapping and absorption optimization in certain thin-film photonic crystal architectures," Phys. Rev. A 78, 023825 (2008).

18. G. Demésy and S. John, "Solar energy trapping with modulated silicon nanowire photonic crystals," J. Appl. Phys. 112, 074326 (2012).

19. S. Eyderman, S. John, and A. Deinega, "Solar light trapping in slanted conical-pore photonic crystals beyond statistical ray trapping," J. Appl. Phys. 113, 154315 (2013).

20. P. Kuang et al., "Light trapping and near-unity solar absorption in a three-dimensional photonic-crystal," Opt. Lett. 38, 4200-4203 (2013).

21. C. Trompoukis et al., "Photonic nanostructures for advanced light trapping in silicon solar cells: the impact of etching on the material electronic quality," Phys. Status Solidi-Rapid Res. Lett. 10, 158-163 (2016).

22. M. J. Kerr and A. Cuevas, "Very low bulk and surface recombination in oxidized silicon wafers," Semicond. Sci. Technol. 17, 35-38 (2001).

23. M. J. Kerr et al., "Surface recombination velocity of phosphorus-diffused silicon solar cell emitters passivated with plasma enhanced chemical vapor deposited silicon nitride and thermal silicon oxide," J. Appl. Phys. 89, 3821-3826 (2001).

24. Z. R. Chowdhury, K. Cho, and N. P. Kherani, "High-quality surface passivation of silicon using native oxide and silicon nitride layers," Appl. Phys. Lett. 101, 021601 (2012).

25. P. Mahtani et al., "High quality amorphous-crystalline silicon heterostructure prepared by grid-biased remote radio-frequency plasma enhanced chemical vapor deposition," J. NonCryst. Solids 358, 3396-3402 (2012).

26. A. Mavrokefalos et al., "Efficient light trapping in inverted nanopyramid thin crystalline silicon membranes for solar cell applications," Nano Lett. 12, 2792-2796 (2012).

27. J. Zhu et al., "Optical absorption enhancement in amorphous silicon nanowire and nanocone arrays," Nano Lett. 9, 279-282 (2009).

28. P. Bermel et al., "Improving thin-film crystalline silicon solar cell efficiencies with photonic crystals," Opt. Express 15, 16986-17000 (2007).

29. S. B. Mallick, M. Agrawal, and P. Peumans, "Optimal light trapping in ultra-thin photonic crystal crystalline silicon solar cells," Opt. Express 18, 5691-5706 (2010).

30. A. Oskooi et al., "Experimental demonstration of quasi-resonant absorption in silicon thin films for enhanced solar light trapping," ACS Photonics 1, 304-309 (2014).

31. J. van de Groep, P. Spinelli, and A. Polman, "Single-step soft-imprinted large-area nanopatterned antireflection coating," Nano Lett. 15, 4223-4228 (2015).

32. C. H. Sun, P. Jiang, and B. Jiang, "Broadband moth-eye antireflection coatings on silicon," Appl. Phys. Lett. 92, 061112 (2008).

33. J.-Q. Xi et al., "Optical thin-film materials with low refractive index for broadband elimination of Fresnel reflection," Nat. Photonics 1, 176-179 (2007).

34. P. Kuang, M.-L. Hsieh, and S.-Y. Lin, "Integrated three-dimensional photonic nanostructures for achieving near-unity solar absorption and superhydrophobicity," J. Appl. Phys. 117, 215309 (2015).

35. M. Chen et al., "Design of optical path for wide-angle gradient-index antireflection coatings," Appl. Opt. 46, 6533-6538 (2007). 
36. R. Legtenberg et al., "Anisotropic reactive ion etching of silicon using $\mathrm{SF}_{6} / \mathrm{O}_{2} / \mathrm{CHF}_{3}$ gas mixtures," J. Electrochem. Soc. 142, 2020-2028 (1995).

37. H. Savin et al., "Black silicon solar cells with interdigitated back-contacts achieve $22.1 \%$ efficiency," Nat. Nanotechnol. 10, 624-628 (2015).

38. K. Seo et al., "Si microwire solar cells: improved efficiency with a conformal $\mathrm{SiO}_{2}$ layer," ACS Nano 7, 5539-5545 (2013).

39. http://fdtd.kintechlab.com/en/download.

40. S. Foster and S. John, "Light-trapping design for thin-film silicon-perovskite tandem solar cells," J. Appl. Phys. 120, 103103 (2016).

41. S. Eyderman, A. Deinega, and S. John, "Near perfect solar absorption in ultra-thin-film GaAs photonic crystals," J. Mater. Chem. A 2, 761-769 (2014).

Ping Kuang received his $\mathrm{PhD}$ from Iowa State University in 2011. He was a research associate at Rensselaer Polytechnic Institute. He is currently a senior etch process engineer at GlobalFoundries. His research interests include plasma etch, photonic crystals, transparent electrodes, and thin-film solar cells.

Sayak Bhattacharya received his $\mathrm{PhD}$ in electrical engineering from Indian Institute of Technology, Delhi, in 2016. He is currently appointed as a postdoctoral fellow in the Department of Physics, University of Toronto. His research interest includes photonic crystals, solar cells, surface plasmon, and periodic structures for spoof surface plasmon at microwave frequencies.

Mei-Li Hsieh received her PhD in the Institute of Electro-Optical Engineering from the National Chiao-Tung University in 2000 and joined the Institute of Electro-Optical Science and Technology in the National Taiwan Normal University as an assistant professor since 2002. From 2011, she joined the Department of Photonics in National Chiao-Tung University as an associate professor. Her research interests include holographic lithography, 3-D holographic display, and optical module and system integration.

Sajeev John is a university professor at the University of Toronto. He received his $\mathrm{PhD}$ in physics at Harvard University in 1984. From 1986 to 1989, he was an assistant professor of physics at Princeton University. While at Princeton, he coinvented the concept of photonic band gap materials in 1987. In the fall of 1989, he joined the senior physics faculty at the University of Toronto.

Shawn-Yu Lin received his $\mathrm{PhD}$ in electrical engineering from Princeton University in 1992. He was at IBM TJ Watson Research Laboratory as a postdoctor fellow, at Sandia National Laboratories as a distinguished member of technical staff and now at Rensselaer Polytechnic as a physics professor. His research interest is in nanophotonics and, in particular, in photonic crystal nanostructures. 